\title{
Mujeres y finanzas en la prensa en línea: análisis desde una perspectiva feminista
}

\section{Women and finance in online print media: analysis from a feminist perspective}

\section{Mulheres e finanças na imprensa escrita online: análise a partir de uma perspectiva feminista}

Lorena Andrea Pérez Roa, Universidad de Chile, Santiago, Chile (loperez@uchile.cl)

Santos Inti Allendes Caro, Universidad de Chile, Santiago, Chile (santos.allendes@ug.uchile.cl)

RESUMEN | Desde un enfoque feminista aplicado a la comunicación, este trabajo explora las representaciones sociales sobre la relación entre mujeres y finanzas en la prensa en línea de Chile. Para ello, se realizó un análisis de contenido cualitativo sobre un corpus de 59 notas de prensa publicadas en periódicos en línea vigentes en Chile. Los resultados presentan dos supuestos dominantes que buscan explicar el comportamiento financiero de las mujeres: el peso de la familia en las decisiones económicas de las mujeres y su comportamiento adverso al riesgo financiero. Se explora cómo estas representaciones sobre las mujeres en las noticias sobre finanzas siguen ancladas a los roles tradicionales de género, sin reflejar la complejidad de la realidad financiera de las mujeres, sus determinantes socioeconómicos y los vínculos entre las finanzas y los nuevos modos de explotación de los cuerpos femeninos.

PALABRAS CLAVES: mujer y finanzas; medios de comunicación y perspectivas feministas; análisis de contenido; medios escritos. 
ABSTRACT | From a feminist approach applied to communication, this paper explores the social representations on the relationship between women and finance in the online press in Chile. For this purpose, we conducted a qualitative content analysis on a corpus of 59 press releases published in online newspapers currently in force in Chile. The results present two assumptions that circulate in the public discussion and that seek to explain women's financial behavior, namely: the weight of the family in women's economic decisions and their financial risk-averse behavior. These assumptions are discussed based on the contributions of a feminist approach applied to communication in order to explore how these representations of women in the financial market remain anchored to traditional gender roles, without reflecting the complexity of women's financial reality, its socioeconomic determinants and the links between finance and the new modes of exploitation of women's bodies.

KEYWORDS: women and finance; media and feminist perspectives; content analysis; print media.

RESUMO|A partir de uma abordagem feminista aplicada à comunicação, este trabalho explora as representações sociais sobre a relação entre as mulheres e as finanças na imprensa online no Chile. Para este efeito, foi realizada uma análise qualitativa do conteúdo de um corpus de 59 comunicados de imprensa publicados em jornais online atualmente em vigor no Chile. Os resultados apresentam dois pressupostos que circulam na discussão pública e que procuram explicar o comportamento financeiro das mulheres, nomeadamente: o peso da família nas decisões econômicas das mulheres e o seu comportamento avesso ao risco financeiro.Explora-se a forma como estas representações das mulheres no mercado financeiro continuam ancoradas nos papéis tradicionais de gênero, sem refletir a complexidade da realidade financeira das mulheres, os seus determinantes socioeconômicos e as ligações entre as finanças e as novas formas de exploração dos corpos das mulheres.

PALAVRAS-CHAVE: mulher e finanças; mídia e perspectivas feministas; análise de conteúdo; mídia escrita. 


\section{INTRODUCCIÓN}

El comportamiento financiero de las mujeres chilenas ha sido monitoreado de cerca por la comisión para el mercado financiero (CMF) desde hace más de una década, mediante el informe de género en el sistema financiero. Este levanta información para el diseño de políticas públicas en materia de equidad de género, dando cuenta de las diferencias entre hombres y mujeres en el acceso a productos de crédito y ahorro. Los resultados del último informe indican que, en Chile, se han logrado disminuir las brechas de género con respecto al acceso y uso de servicios financieros (Comisión para el mercado financiero, 2020). En relación con el comportamiento financiero, si bien el de las mujeres es sistemáticamente mejor que el de los hombres, el mercado sigue confiando mayores montos y mejores condiciones crediticias a estos últimos.

Las diferencias salariales y las dificultades de acceso al trabajo remunerado son elementos clave para explicar la brecha de género en el sistema financiero. En efecto, la brecha salarial en Chile según la Encuesta Suplementaria de Ingresos 2018 estima que las mujeres perciben un 27,2\% menos de ingresos en comparación con los hombres. Por ello las mujeres tendrían una baja tasa de soporte y un acceso limitado a productos financieros, peores condiciones crediticias y una mayor probabilidad de endeudamiento (Comisión para el mercado financiero, 2018).

La evidencia ha insistido en la importancia de considerar los sesgos de género en la socialización financiera para entender las brechas de acceso (Agnew et al., 2018). Estos sesgos, que comenzarían a temprana edad (Rudeloff et al., 2019), tienden a vincular a las mujeres con las prácticas de ahorro, excluyéndolas de las discusiones económicas (Yu et al., 2015). En el caso de los varones, se les enseña a confiar, desde temprana edad, en sus habilidades económicas, involucrándolos activamente en las discusiones financieras (Al-Bahrani et al., 2020).

La prensa escrita, en tanto agente de socialización (Calvo Ortega, 2014), adquiere una relevancia estratégica en el abordaje de estos debates, problematizando los roles tradicionales de género que se reproducen en el espacio financiero. Entendiendo que la prensa tiene la capacidad de construir una agenda informativa que impacte en el debate público (Gronemeyer \& Porath, 2017), consideramos relevante explorar las representaciones de las mujeres en el sistema financiero en este espacio informativo. Los medios de comunicación participan de la reproducción de estereotipos en la sociedad (Puleo, 2007; García Beaudoux et al., 2018), amplificando una serie de creencias y lugares comunes respecto de cómo deberían manejar sus conductas las personas generizadas (Huici et al., 2006). Un mayor tratamiento informativo de estos temas podría incidir en la realidad vital de las lectoras y proporcionarles mejores elementos para evaluar los informes financieros (Rovetto, 2010). 
En la línea de los trabajos de Federici y sus colegas (2018), Adkins (2018) y Cavallero y Gago (2019), consideramos que una aproximación feminista sobre las prácticas financieras juega un rol formulador de estructuras sociales y morales, tradicionalmente forjadas desde una idea de lo económico distante de las mujeres como sujetos, a partir de un modelo de familia heteronormado. Así, se podría representar de mejor manera a las mujeres en el discurso informativo (Rovetto, 2010) y promover aproximaciones a la economía más cercanas a su realidad.

En la línea de los trabajos de Lee $(2014,2015,2019)$ quien recurre a un enfoque feminista aplicado a la comunicación, se explora cómo la prensa puede jugar un rol estabilizador de las relaciones sociales desiguales de género al informar sobre los mercados financieros. En este sentido, la prensa se entiende como un "lugar de producción de conocimiento" (Lee, 2014, p. 273), es decir, se asume que los discursos mediáticos son un factor importante a la hora de elaborar estereotipos.

\section{La crítica feminista a las representaciones de género en los medios}

La crítica feminista en los medios lleva décadas construyendo una agenda de investigación sobre la necesidad de una representación de las mujeres más acorde con las transformaciones sociales contemporáneas (Rovetto, 2010). Esta perspectiva se articula a partir de tres ámbitos de investigación: 1) la estructura organizacional, que estudia el dominio del liderazgo masculino en la industria; 2) la emisión de mensajes, que analiza la representación de las mujeres en el discurso mediático, y 3) la recepción de contenidos, que comprende la audiencia femenina (Vega Montiel, en Rovetto, 2013). La evidencia enfatiza la relevancia que tienen los medios a la hora de elaborar estereotipos, representaciones e imaginarios dominantes de género. Se ha estudiado el uso de las fuentes informativas como un indicador de la discriminación de género en las redacciones (Rovetto \& Figueroa, 2019). Para las autoras, la ausencia de una perspectiva de género es una "expresión simbólica del sexismo en el discurso informativo y el uso de fuentes funciona como un indicador de la discriminación de género en las redacciones" (p.63).

Si bien el análisis de las representaciones financieras desde una perspectiva feminista en los medios de comunicación ha sido poco abordado, los trabajos desarrollados por Lee $(2014,2019)$ buscan contribuir a una agenda de investigación en esta área. En sus últimos trabajos, la autora explora la representación mediática de la relación entre las mujeres y las finanzas. Específicamente, analiza los libros de autoayuda dirigidos a mujeres (2014) y las películas de ficción de Hollywood sobre los mercados financieros $(2014,2019)$. Su interés es dar cuenta sobre cómo estas representaciones de género obscurecen el trabajo de reproducción social y promueven una mercantilización de las capacidades corporales de las mujeres. 
Para ello, la autora propone el desarrollo de un enfoque económico-político y feminista de la comunicación que permita examinar tanto la producción, distribución y consumo de bienes en función del género, como la utilización de ideologías para estabilizar las relaciones sociales desiguales. Esta perspectiva parte del supuesto de que los medios de comunicación forman parte integral de los procesos económicos, políticos, sociales y culturales fundamentales de una sociedad (Mosco, 2009, en Lee, 2015). A su juicio, es un imperativo disciplinar promover una aproximación feminista al análisis de la intersección entre género, finanzas y medios de comunicación desde los discursos populares, para desenmascarar las dimensiones macroeconómicas ocultas entre el género y las finanzas y reparar lo que denomina "la grieta entre la investigación micro y macroeconómica que tradicionalmente nos presentan las finanzas como áreas desconectadas y descorporalizadas" (Lee, 2014).

\section{La crítica feminista a la representación de género en las finanzas}

La crítica feminista en el espacio financiero se articula a partir de la denuncia al sesgo androcéntrico que le atribuye al homo economicus características que considera universales para la especie humana, pero que son propias de un ser humano "varón, blanco, adulto, heterosexual, sano, de ingresos medios" (Rodríguez Enríquez, 2015). De esta manera, busca dar cuenta críticamente de los supuestos políticos-culturales que organizan la actividad económica y que han relegado a las mujeres a un espacio subalterno (Pérez Orozco, 2004). Específicamente, cuestiona el rol asignado a las mujeres en el espacio doméstico, enfocando su acción económica en el cuidado y bienestar familiar, enfatizando sus capacidades reproductivas y la dependencia de los hombres del núcleo familiar.

A partir de la crisis financiera global de 2008, la crítica feminista comenzó a volcar su interés en las finanzas y en el análisis de las narrativas que explicaron la crisis financiera global. Hozic y True (2016) reúnen una serie de trabajos sobre la crisis desde sus dimensiones de género, abordando las raíces estructurales de la desigualdad, la exclusión y la opresión de las estructuras financieras. Su propósito es criticar los aspectos de la economía que afectan el bienestar, pero que tienden a ser ignorados en los análisis macroeconómicos, y cuestionar, a su vez, la narrativa punitiva que instala la idea de que la crisis resulta de una serie de acontecimientos contagiosos exógenos al sistema capitalista y que deben manejarse a través de la contención punitiva de los culpables (Cameron et al., 2011; Brassett \& Tehel, 2015; Hozic \& True, 2016). Otro punto relevante de esta línea de trabajo es la denuncia a las nuevas formas de explotación que exponen a los grupos marginados, como las mujeres, personas racializadas y migrantes a productos financieros de mayor riesgo e inestabilidad (Adkins, 2018). 
En esta línea, Roberts $(2015,2016)$ identifica tres relatos presentes en las instituciones internacionales (FMI, Banco Mundial, Naciones Unidas, OCDE, entre otras) que buscan impulsar intervenciones en el mercado financiero a favor de las mujeres. La primera narrativa refiere a la idea de la mujer como salvadora, que afirma que una mayor integración en los mercados financieros, específicamente de las mujeres del Sur Global, ayudará a aliviar la pobreza y estimular las economías nacionales. El segundo relato refiere a la igualdad tecnocrática, que sostiene que las nuevas tecnologías de las finanzas han ayudado a eliminar la discriminación basada en género, al estandarizar los modelos de evaluación del riesgo. El tercer relato, es el de las womeconomics, que insiste en las ventajas o dividendos de género que pueden obtenerse al promover que asuman cargos más altos en las instituciones financieras. Estos tres relatos, a juicio de esta autora, producen un argumento comercial en favor del género, que busca aumentar la participación de las mujeres en los mercados financieros y mejorar su capacidad de consumir bienes y servicios. En este sentido, son relatos que entienden las desigualdades de género como una imperfección del mercado y no como una estructura condicionada por las relaciones de poder-producción y reproducción social de los mercados financieros.

\section{METODOLOGÍA}

El objetivo de esta investigación es explorar, desde un enfoque feminista aplicado a la comunicación, las representaciones sociales sobre la relación entre mujeres y finanzas en la prensa en línea de Chile. El trabajo pone el foco en los procesos de creación y emisión de representaciones vinculadas con las mujeres en los medios informativos (Gonem \& i Solà, 2009). Para ello, se realizó un análisis de contenido cualitativo sobre un corpus de 59 notas de prensa publicadas en periódicos en línea de Chile.

Entenderemos por prensa en línea al conjunto de medios escritos que digitalizan su contenido en plataformas web (Díaz \& Mellado, 2017). La prensa en formato digital ha asumido gran protagonismo en la última década, posicionándose como un espacio de interacción, e intervención para los lectores (Raimondo Anselmino, 2014; Gascón, 2020). Esta actividad deja huella en la interfaz del periódico, permite rankings de lectura, comentarios o encuestas, entre otros (Raimondo Anselmino, 2012). Por otro lado, las representaciones sociales presentes en la prensa en línea se ponen a disposición de una audiencia cada vez más amplia y participativa. Sin embargo, la información digitalizada no es neutral. Los diarios tienen la facultad de dotar de importancia a ciertas temáticas, y negarles visibilidad a otras (Díaz \& Mellado, 2017; McCombs \& Valenzuela, 2007). En este sentido, y considerando 
que los procesos de emisión de noticias están permeados por representaciones de género, nuestro trabajo busca explorar las construidas cuando se informa sobre las mujeres en el ámbito financiero, analizando la emisión de repeticiones sociales vinculadas con las mujeres (Gonem \& i Solà, 2009), para contribuir a escenarios comunicativos más integradores e igualitarios en materia de género (Rovetto \& Simelio, 2012).

Las notas de prensa analizadas fueron publicadas entre los años 2014 y 2019. La elección de este período responde a que, a lo largo de esos años, se comenzaron a publicar los informes Género en el sistema financiero e Informe de Deuda Morosa, por parte de la Comisión para los Mercados Financieros y Equifax, en alianza con la Universidad San Sebastián, respectivamente. El primer informe surge de un compromiso institucional por generar información que aporte a diseñar políticas públicas para superar las brechas de género en el sistema financiero. En lo que respecta al segundo, realiza un análisis técnico de la evolución de las deudas impagas de créditos personales. Se caracterizan por publicar una batería de datos sobre el acceso y el uso de productos de crédito en Chile, desagregados según género, dando cuenta así de las profundas desigualdades que atraviesan al sistema financiero chileno en la materia.

En la tabla 1 se presenta un resumen de los medios y de la proporción de notas que publicaron información sobre ambos informes durante el período estudiado.

Este corpus se obtuvo a partir de una búsqueda de las notas de prensa que hicieran una referencia directa a los resultados de estos estudios y elaboraran una interpretación de estos en los sitios web de los periódicos. La mayoría de estas se publicaron semanas después de haber sido difundidos los resultados de ambos informes. Cabe destacar que el informe de género en el sistema financiero se publica anualmente y que el de deuda morosa es trimestral.

Se realizó un análisis de contenido cualitativo. Esta técnica consiste en interpretar los mensajes que contienen determinados textos, discursos, o productos comunicativos (Piñuel, 2002) para develar aspectos específicos de un fenómeno que se manifiesta en un determinado contexto. En este sentido, es una herramienta útil para reinterpretar el contenido manifiesto de los textos, de modo de generar nuevas lecturas sobre el fenómeno investigado (Wimmer \& Dominik, 2001). El proceso de análisis se estructuró en dos momentos. Primero, se realizó un proceso de categorización inductiva (Álvarez, 2012), donde el objeto de análisis fue el cuerpo de la noticia. Así, se buscó reducir el volumen de la información y categorizarla en función de las principales representaciones culturales sobre la relación entre mujeres y finanzas. Luego, se da cuenta del contenido de algunas de las representaciones encontradas en ambas categorías. 
Diario en línea

\begin{tabular}{cc}
\hline ADN Radio & 1 \\
\hline Atacama Noticias & 1 \\
\hline Bio - Bio & 4 \\
\hline Diario Concepción & 2 \\
\hline Diario Financiero & 2 \\
\hline Diario La Estrategia & 1 \\
\hline El Desconcierto & 1 \\
\hline El Dínamo & 3 \\
\hline El Mostrador & 8 \\
\hline El Tipógrafo & 1 \\
\hline EMOL & 3 \\
\hline Gran Valparaíso & 1 \\
\hline La Cuarta & 1 \\
\hline La Segunda Móvil & 1 \\
\hline La Tercera & 23 \\
\hline Publimetro & 4 \\
\hline Tarapacá online & 1 \\
\hline The Clinic & 1 \\
\hline Total & 59 \\
\hline
\end{tabular}

Tabla 1. Cantidad de notas de prensa según diario digital (2014-2019)

Fuente: Elaboración propia.

\section{Informes}

\begin{tabular}{cccc}
\cline { 2 - 4 } Añ $^{*}$ & Género en sistema financiero & Informe de deuda morosa & Otros informes** \\
\hline 2014 & 2 & 1 & 0 \\
\hline 2015 & 2 & 1 & 2 \\
\hline 2016 & 2 & 2 & 0 \\
\hline 2017 & 6 & 1 & 6 \\
\hline 2018 & 12 & 7 & 2 \\
\hline 2019 & 4 & 8 & 1 \\
\hline Total & 28 & 20 & 11 \\
\hline
\end{tabular}

* La tabla busca dar cuenta de la cantidad de notas de prensa que se publicaron en el periodo 2014 - 2018.

** Estos informes son publicados en su mayoría por CHILEDEUDAS, una organización jurídica que ha realizado estudios, y trabajos de difusión periodista en función de los resultados del informe de deuda morosa, y género en el sistema financiero.

Tabla 2. Cantidad de notas de prensa seleccionadas que refieren a los informes

Fuente: Elaboración propia. 


\section{RESULTADOS}

Del proceso se categorización inductiva se levantaron dos encuadres dominantes:

- Familia primero: hace referencia a la relación entre el comportamiento financiero de las mujeres y el progreso del hogar.

- Adversidad al riesgo: remite a los comportamientos financieros más conservadores por parte de las mujeres.

\section{La familia primero: mujeres y economía doméstica}

Una de las imágenes que circulan con mayor fuerza en la prensa escrita sobre la relación de las mujeres con las finanzas, es su vínculo casi natural con una buena administración del hogar. En efecto, de las 59 notas en línea revisadas, 30\% se refiere a las mujeres en el espacio financiero aludiendo explícitamente a sus capacidades para administrar el hogar. Estas habilidades se expresan en el espacio de la prensa en línea principalmente de dos maneras: 1) como una segmentación del mercado, es decir, como consumidoras preferenciales de productos financieros pensados para ellas y la protección de la familia, y 2) como una conducta castigada por el sistema financiero, que finaliza por marginalizarlas de sus beneficios. Cabe destacar que, del total de notas de prensa vinculada a la categoría la familia primero, $35 \%$ hace referencia a la segmentación del mercado y $65 \%$, a la conducta castigada. Este encuadre se puede ilustrar con el siguiente ejemplo:

Responde honestamente: ¿Quién en tu familia decide cuánto gastar en la compra semanal, ahorra dinero progresivamente, busca precios hasta encontrar el mejor servicio, o compara distintas cuotas bancarias para obtener las mejores condiciones? Es muy probable que la persona que te haya venido a la cabeza sea tu madre (e incluso tú, si eres mujer) (Yasinetskaya, 2017).

En relación con la segmentación del mercado para responder a las necesidades de las mujeres, gran parte de los contenidos analizados usan los comportamientos atribuidos tradicionalmente a las mujeres como una explicación disponible para analizar los datos emitidos por los informes Género en el Sistema Financiero (Comisión para el mercado financieroMF):

Las chilenas, de acuerdo con el informe, presentan una demanda más activa que los hombres en productos de ahorro y en deuda para una vivienda. Es decir, pareciera que la mujer se endeuda más con una visión a futuro y buscando la estabilidad del núcleo familiar (“Las mujeres son menos morosas pero...", 2018).

Si bien el informe intenciona en sus análisis las diferencias entre el comportamiento de las mujeres y los hombres, pues su objetivo es superar las brechas de género en el sistema financiero, suponer que ahorran para ser dueñas de 
su hogar o que buscan la estabilidad familiar implica presumir la voluntad colectiva de los proyectos económicos femeninos. Así, por ejemplo, en otro reportaje realizado por el mismo diario, referido a la contratación de seguros privados por parte de las mujeres, se indica el aumento de los hogares con jefaturas femininas, sus lagunas provisionales y la maternidad, tres características de segmentación que el mercado de seguros utiliza para ofrecerles sus servicios:

El aumento de jefas de hogares que creció de $29,3 \%$ a 37,6\% en el período 2006-2015, según el INE, hecho que las impulsara a contratar seguros que respondan a la protección para sus hijos (...) A la hora de contratar seguros -las mujeres- lo harían en función de su edad, donde de 20 a 35 años sus preferencias irían ligadas a los seguros de salud, seguro con APV, enfocado en mejorar su pensión y seguros de vida como medio de protección para sus hijos. Otro de los factores del alza de demanda de seguros estaría ligado a razones de maternidad, lagunas previsionales, con el fin de no solo aumentar la pensión, sino que tener una protección financiera para el hogar "Contratación de seguros con ahorro por mujeres...", 2018).

A pesar de que las noticias analizadas asumen el rol del mercado financiero en la generación de respuestas a las desigualdades estructurales que afectan particularmente a las mujeres, los resultados sí dan cuenta de una preocupación por sus condiciones materiales de vida. Esto se observa particularmente en las notas de prensa referidas a los resultados de los informes de morosidad. Un $36 \%$ de estas cuestiona la situación financiera que las afecta. Por ejemplo, en la nota de prensa ¿Por quélos chilenos se endeudan tanto? se plantea explícitamente esta preocupación:

Dada la proporción de mujeres con cuotas impagas [deudas morosas], se puede plantear la hipótesis que tienen acceso a créditos un porcentaje de ellas con ingresos bajos o nulos, en emisores de crédito que no son bancos" La delicada situación económica del país se está evidenciando en estas cifras, en donde sectores más sensibles a los ciclos económicos, como lo son jóvenes, adultos mayores y mujeres, están revelando una situación más estrecha. Esto no solo por las cifras de morosidad propiamente tal, sino por las circunstancias que hay detrás de ellas; tales como niveles de ingreso, tasa de desempleo y las tasas de inflación, que han deteriorado la capacidad de pago de los segmentos más vulnerables (“76\% de los deudores morosos...", 2015).

A pesar de lo anterior, las noticias analizadas no profundizan en cómo la condición femenina es abordada por el sistema en códigos de riesgo financiero. Tal como lo señalan las siguientes notas de prensa, las mujeres son castigadas por el sistema financiero por ser mujeres, madres y responsables del bienestar familiar: 
Las que se encuentran en edad fértil, esto es, entre los 25 y 40 años, se les cobran intereses más altos por un crédito bancario. Lo anterior, dado que, para las entidades financieras, su riesgo es menor, porque para dichas empresas, cuando una mujer queda embarazada probablemente dejará de trabajar y perderá su solvencia económica, a pesar de que las mujeres son mejores pagadoras que los hombres y ahorran más que ellos. Los intereses son hasta 20\% mayores en el rango de edad 25-40 años, en comparación al rango de edad 40-50 años (Montes, 2019).

En su racionalidad económica, las entidades financieras pueden estar aplicando condiciones de plazo, monto y/o tasas más restrictivas a individuos o grupos percibidos como más riesgosos. En tal ámbito las brechas de ingresos, la falta de historial crediticio y las condiciones de estabilidad laboral podrían estar afectando el acceso a crédito de las mujeres" (Arroyo, 2017).

Todos estos castigos (edad fértil, brecha salarial e inestabilidad laboral) se traducen en peores condiciones de acceso al crédito; tasas de interés más elevadas, acceso a menores montos de crédito. La transversalidad de la inequidad de género cruza también la dimensión financiera y -al igual que lo que sucede en otros espacios- son discriminaciones de género que no tienen argumentos técnicos para justificarse.

\section{La adversidad de las mujeres al riesgo: ahorradoras, pagadoras, pero conservadoras.}

Los estereotipos de género para explicar las condiciones crediticias desfavorables que el mercado financiero les ofrece a las mujeres son reproducidos en las noticias analizadas. El mercado financiero respondería en concordancia al comportamiento natural de las mujeres, quienes son representadas como: ahorrativas, buenas pagadoras y adversas al riesgo financiero. El 29\% de las notas de prensa oscila entre estos tres ejes interpretativos y ocupa estos términos para explicar su comportamiento financiero. La referencia más frecuente es que son buenas pagadoras (70\%), seguido por la idea de las mujeres como ahorrativas (17\%) y adversas a los riesgos financieros (13\%).

La cualidad de ahorrativas y buenas pagadoras van generalmente de la mano: "Las mujeres son mejores pagadoras y ahorran más, por lo tanto, caen menos en morosidad que los hombres, al ser más ordenadas en términos financieros" (Montes, 2019); "Las mujeres son mejores pagadoras que los hombres chilenos, mostrando una mayor responsabilidad financiera que la de ellos" ("A la hora de la calillas...", 2019). Efectivamente, los productos de ahorro contratados por las mujeres han aumentado de manera significativa en comparación con los hombres (Comisión de mercados financieros, 2018). Ahora bien, que las mujeres tengan más productos 
de ahorro no implica necesariamente que puedan ahorrar más, pues sus montos de ahorro son inferiores. En este sentido, las diferencia salariales condicionan las posibilidades de ahorro de las mujeres. A pesar de ello, las noticias insisten en vincular las conductas de ahorro con la adversión al riesgo: "Las mujeres son más conservadoras que los hombres al momento de tomar créditos, lo que se confirma también en que ahorran 20\% más" (“A la hora de las calillas”, 2019).

Finalmente, sobre la habilidad de las mujeres de responder a tiempo a los compromisos financieros, creemos relevante detallar dos elementos. En primer lugar, enfatizar que la información entregada por los informes financieros da cuenta de que las deudas de las mujeres son por lo general más pequeñas y están especialmente concentradas en el retail. Lo anterior implica que las mujeres acceden, por lo general, a créditos con montos menores, pero con tasas de interés más elevadas. Según lo informado por la prensa, esta tendencia se mantiene desde 2012. En segundo lugar, enfatizar que, a pesar de que existen datos objetivos que muestran que las mujeres tienden a responder en los plazos sus compromisos financieros, como que presentan "menos cheques protestados" y "menos tarjetas de crédito con morosidad", la morosidad femenina ha ido aumentando en los últimos años. Esto, a pesar de que el monto de mora de los hombres sigue siendo casi el doble que el de las mujeres.

En este sentido, los últimos informes de Equifax (Equifax, 2019) no entregan evidencia contundente de que las mujeres sean mejores o peores pagadoras que los hombres, pero sí dan cuenta que están en una posición más frágil para cumplir sus compromisos, debido a lo sensible que es esta conducta a las condiciones salariales. Es decir, la condición de buena pagadora depende de las posibilidades de pago. En este sentido, vale la pena preguntarse si las mujeres podrán mantener este atributo de buenas pagadoras si sus condiciones salariales se precarizan aún más. De ahí la relevancia de observar críticamente el llamado a superar las brechas de género y favorecer la profundización financiera, ya que esta se construye con base en desigualdades estructurales que pueden acentuarse si se buscan superarlas a tavés de deudas.

\section{DISCUSIÓN}

Los hallazgos indican dos grandes representaciones de la relación entre mujeres y finanzas que circulan en la prensa en línea chilena: 1) el peso de la familia en sus decisiones económicas y 2) la persistencia de un comportamiento adverso al riesgo financiero. 
Con respecto al primer supuesto, nuestro trabajo constató dos maneras distintas en que lo expresan los periodistas. Primero, como un proceso de diversificación financiera que crea nuevos productos pensados para mujeres, con el objeto de sopesar las desigualdades económicas a las que se enfrentan (lagunas previsionales, seguros para la educación de hijos/as, etc.) o para financiar las actividades de reproducción social (créditos para dueñas de casa, microcréditos, entre otros). Segundo, como una conducta castigada por el sistema , que tiende a marginalizar a las mujeres de sus beneficios económicos.

Con respecto a los procesos de diversificación financiera y de creación de mercados especialmente pensados para mujeres, podemos dar cuenta de que estos tienden a naturalizar las desigualdades estructurales de género existentes, promoviendo un modelo de inclusión de las mujeres al sistema financiero en el que se mercantilizan las desigualdades económicas, creando nuevos nichos de negocios de mayor riesgo e inestabilidad (Adkins, 2018). Las lagunas previsionales por maternidad y el mandato de proteger el futuro económico de los hijos no son ni responsabilidades de las mujeres ni fallas del mercado, sino que responderían a estructuras de género que sostienen los paradigmas dominantes de las finanzas y la gobernanza mundial (Hozic \& True, 2016). Entendiendo la importancia que tienen las representaciones de género difundidas en la prensa estudiada, consideramos relevante profundizar en estas condiciones estructurales y evitar naturalizar las condiciones financieras de las mujeres como un hecho de exclusiva responsabilidad individual.

Sobre la representación de las finanzas como un espacio legítimo para responder a las necesidades de reproducción social y entendiendo la posición central en esta tarea que históricamente han ocupado las mujeres, no es de extrañar que el sistema financiero ofrezca respuestas específicas para que asuman los riesgos familiares. Si bien diversos trabajos de investigación realizados en Chile ofrecen evidencia empírica sobre las formas en que la reproducción social esta cada vez más estructuralmente vinculada a las finanzas (Pérez Roa, 2020; López \& Bonilla, 2020), la estrategia de favorecer la integración de las mujeres a los mercados financieros es considerada una forma de explotación financiera (Cavallero \& Gago, 2019). Las finanzas construyen argumentos comerciales (Roberts, 2015) ligados a los roles tradicionales de cuidado y protección familiar para promover el consumo de productos financieros, comprometiendo así su futura capacidad de pago (Federici et al., 2018). En este sentido, las notas de prensa analizadas dan cuenta cómo las finanzas construyen un argumento comercial en razón de la propia condición de mujer, promoviendo así una mercantilización de sus capacidades corporales (Lee, 2015). 
En relación con los comportamientos de ahorro y de pago, y la supuesta adversidad de las mujeres frente al riesgo financiero, nuestro análisis reconoce tres elementos en los reportajes de prensa analizados. Primero, las noticias tienden a establecer un vínculo entre la predisposición al ahorro y los buenos comportamientos de pago de las mujeres; segundo, estos comportamientos de ahorro y pago se asocian a la protección familiar futura y, tercero, se asume que las mujeres son naturalmente adversas al riesgo.

Acerca de las conductas financieras, observamos cómo se representa el ahorro de las mujeres vinculado naturalmente a la previsión familiar. Sin embargo, esta idea responde a una concepción limitada de los ahorros de las mujeres. Diversos estudios (Pérez-Roa \& Troncoso, 2019; Belleau \& Lobet, 2017; Zelizer, 2011) discuten cómo el ahorro es una práctica recurrente en las mujeres a través del tiempo, en razón de las dificultades históricas de acceso al dinero: mujeres que acceden al él a través de otros (Belleau \& Lobet, 2017), prevalencia femenina en el mercado informal (Villarreal, 2008), transferencias económicas entre redes de apoyo (PérezRoa, 2019) y las brechas salariales persistentes en el mercado del trabajo salariado. Ahora bien, etiquetar las prácticas de ahorro como una adversión al riesgo favorece una lectura androcéntrica de las finanzas, colonizada por un ideal de un hombre blanco, adulto, heterosexual que asume riesgos sometiéndose a la lógica de acumulación de capital que las finanzas exigen (Rodríguez Enríquez, 2015). En otras palabras, que el riesgo se represente como una virtud financiera en la prensa responde a la idea de que los hombres se mueven por motivos egoístas, mientras que las mujeres lo hacen por comportamientos altruistas, buscando privilegiar el bienestar de sus hogares por sobre el propio. Estos supuestos han organizado la actividad económica y relegado a las mujeres a un espacio subalterno (Hartmann \& Folbre en Orozco, 2004). Así, al etiquetar las conductas financieras arriesgadas como conductas positivas para el mercado financiero, se desconoce a las mujeres en un doble sentido: primero, les niega la posibilidad de ser reconocida como una persona con autoridad en el mercado financiero (Lee \& Raesch, 2014) y segundo, favorece una conceptualización de ellas como víctimas de la economía mundial masculina hecha para hombres (Lee, 2014), desconociendo que las relaciones de género son construcciones sociales e históricas y no una mera características de los individuos (Hozic \& True, 2016).

\section{CONCLUSIONES}

Las representaciones referidas a la relación entre mujeres y finanzas en la prensa chilena siguen ancladas a sus roles tradicionales en el espacio económico, sin reflejar la complejidad de su realidad financiera, sus determinantes socioeconómicos y los 
vínculos entre las finanzas y los nuevos modos de explotación de los cuerpos femeninos. Lo anterior invita a avanzar hacia un análisis que vincule los problemas financieros de las mujeres con una lectura que problematice el lugar subalterno que han ocupado en el mundo de la economía y las finanzas. En este sentido, más que idealizar los comportamientos de las mujeres con frases del tipo "las mujeres son buenas pagadoras", o "siempre velan por el interés de la familia", hay que abordar esta relación como el resultado de una trama más compleja de relaciones de poder que han obstaculizado su acceso a los recursos económicos (Federici, 2014; Roberts, 2015; Adkins, 2018).

En un contexto de crisis económica y sanitaria, donde los indicadores chilenos de progreso de la situación económica de las mujeres retrocedieron, es aún más relevante favorecer una perspectiva feminista para abordar la relación entre mujeres y finanzas en el periodismo. Su tasa de participación laboral retrocedió más de 10 años -de 47\% en 2010 a 42,9\% , según Mizala (2020). El Instituto Nacional de Estadísticas estima que alrededor de 910.000 mujeres han perdido sus empleos en Chile durante esta pandemia (Mendía \& Pérez, 2020) y 83\% no logra cubrir sus deudas con sus ingresos (Placencia, 2020). Adoptar una postura crítica implica problematizar el supuesto extendido de que las finanzas son una estrategia efectiva y neutral para resolver las brechas de género (Lee, 2019).

\section{FINANCIAMIENTO}

Elartículo fue posiblegracias alfinanciamiento del proyecto "Deudas, mujeres y programas sociales: Explorando el lugar de las mujeres en los programas de educación financiera", Concurso Fortalecimiento de Productividad y Continuidad en Investigación (FPCI- N²1- 0419), de la Facultad de Ciencias Sociales de la Universidad de Chile. También se benefició de recursos del Programa Iniciativa científica Milenio de ANID (Agencia Nacional de Investigación y Desarrollo de Chile), adjudicado al Centro Núcleo Milenio Autoridad y Asimetrías de Poder (numaap.cl)- (ANID Milenio NCS17_ 007 2018).

\section{REFERENCIAS}

Adkins, L. (2018). Money: A feminist issue. Australian Feminist Studies, 33(96), 167-171. https://doi.org/10.1080/08164649.2018.1517253

Agnew, S., Maras, P., \& Moon, A. (2018). Gender differences in financial socialization in the home-An exploratory study. International journal of consumer studies, 42 (3), 275-282. https://doi.org/10.1111/ijcs.12415 
Al-Bahrani, A., Buser, W., \& Patel, D (2020). Early Causes of Financial Disquiet and the Gender Gap in Financial Literacy: Evidence from College Students in the Southeastern United States. Journal of Family and Economic Issues, 41(3), 558-571 https://doi.org/10.1007/s10834-020-09670-3

Álvarez, A. (2012). Redefinición de la radio universitaria en el ámbito virtual (Redefinition of the university radio in the virtual field) (Master's thesis). Retrieved from http://noesis.uis.edu.co/bitstream/123456789/32369/1/143020.pdf

Arroyo, C. (10 de enero, 2018). Mujeres acceden a menores créditos y a mayores tasas que los hombres (Women have access to less credit and at higher rates than men). La Tercera. https://www.latercera.com/noticia/mujeres-acceden-menores-creditos-mayores-tasaslos-hombres/

Belleau, H \& Lobet, D. (2017). L'amour et l'argent. Les éditions du remue-ménage.

Bohórquez-Pereira, G., Espinel Rubio, G. A., \& Mojica Acevedo, E. C. (2020). Mujeres columnistas, liderazgos de opinión en un medio en línea colombiano (Women columnists, opinion leaders in a Colombian online media). Cuadernos.info, (46), 66-95. https://doi.org/10.7764/cdi.46.1731

Brassett, J. \& Rethel, L. (2015). Sexy Money: e Hetero-Normative Politics of Global Finance. Review of International Studies, 41(3), 429-449. https://doi.org/10.1017/S0260210514000461

Calvo Ortega, E. (2014). La representación de la mujer y los roles de género en los informativos deportivos de televisión (The representation of women and gender roles in sports television news). Fonseca, Journal of Communication, 8(8), 111-129. Retrieved from https://revistas.usal.es/index.php/2172-9077/article/view/11814

Cameron, A., Nesvetailova, A., \& Palan, R. (2011). Wages of Sin? Crisis and the Libidinal Economy. Journal of Cultural Economy, 4(2), 117-135. https://doi.org/10.1080/17530350.2011.563066

Cavallero, L. \& Gago, V. (2019). Una lectura feminista de la deuda: "Vivas, libres y desendeudadas nos queremos" (A feminist reading of the debt: "We want to be alive, free and debt-free). Fundación Rosa Luxemburgo. Retrieved from https://rosalux-ba.org/wp-content/ uploads/2019/05/lectura-feminista-deuda-PANTALLAS.pdf

Comisión para el mercado financiero (2018). Género en el sistema financiero (Gender in the financial system). Santiago, Chile. Retrieved from

https://financialallianceforwomen.org/download/genero-en-el-sistema-financiero-2018/

Comisión para el mercado financiero (2020). Género en el sistema financiero (Gender in the financial system). Santiago, Chile. Retrieved from https://www.cmfchile.cl/portal/estadisticas/617/articles-38741_recurso_1.pdf

Díaz, M. \& Mellado, C. (2017). Agenda y uso de fuentes en los titulares y noticias centrales de los medios informativos chilenos. Un estudio de la prensa impresa, online, radio y televisión (Agenda and use of sources in headlines and lead stories in Chilean media. A study of the press, online, radio and television news). Cuadernos.info, (40), 107-121. http://doi.org/10.7764/cdi.40.1106

EQUIFAX. (2020).XXVII Informe de Deuda Morosa (XXVII Debt Report). Retrieved from https://www.uss.cl/wp-content/uploads/2020/01/XXVII-Informe-de-Deuda-Morosa.pdf 
Federici, S., Guervós, C. F., \& Ponz, P. M. (2018). El patriarcado del salario: Críticas feministas al marxismo (The patriarchy of the wage: Feminist critiques on Marxism). Traficantes de sueños.

García Beaudoux, V., D’Adamo, O., \& Gavensky, M. (2018). Una tipología de los sesgos y estereotipos de género en la cobertura periodística de las mujeres candidatas (A Typology of Gender Biases and Stereotypes in the Journalistic Coverage of Women Candidates). Revista Mexicana de Opinión Pública, (24), 113-129. http://doi.org/10.22201/ fcpys.24484911e.2018.24.61614

Gascón, J. F. F., Vila, F., Rodríguez, J. R., \& Sobrepere, M. P. (2020). Interactividad en la prensa online. Una comparativa en diarios regionales y de referencia en Cataluña (Interactivity in the online press. A comparison of regional and reference newspapers in Catalonia). Estudios sobre el mensaje periodístico, 26(4), 1421-1431. https://doi.org/10.5209/esmp.67033

Gonem, F. R. \& i Solà, N. S. (2009). La representación de las mujeres trabajadoras en la prensa española (The representation of working women in the Spanish press). Cuestiones de género: de la igualdad y la diferencia, 4, 193-216. http://doi.org/10.18002/cg.v0i4.3812

Gronemeyer, M. E. \& Porath, W. (2017). Tendencias de la posición editorial en diarios de referencia en Chile: El arte de dosificar la crítica frente a la actuación de los actores políticos (Trends of the editorial viewpoints in reference newspapers in Chile: The art of balancing criticism of the performance of political actors). Revista de Ciencia Politica (Santiago), 37(1), 177-202. http://doi.org/10.4067/S0718-090X2017000100008

Hozic, A. A. \& True, J. (Eds.). (2016). Scandalous economics: Gender and the politics of financial crises. Oxford University Press.

Huici, C., López, A. B., \& Alonso, F. M. (Coords.). (2006). Psicología de grupos (Group psychology). Universidad Nacional de Educación a Distancia, UNED.

La Cuarta (11 de agosto 2019). A la hora de las calillas, somos "chilenes" (When it comes to “calillas", we are "chilenes"). La Cuarta. https://www.lacuarta.com/cronica/noticia/lahora-de-las-calillas-somos-chilenes/396663/

La Tercera (2018). Las mujeres son menos morosas pero tienen más dificultades para acceder a créditos (Women are less delinquent but have more difficulties in accessing credit). La Tercera.https://www.latercera.com/nacional/noticia/viainclusiva-las-mujeres-menosmorosas-tienen-mas-dificultades-acceder-creditos/320072/

La Tercera (22 de mayo 2018 ). Contratación de seguros con ahorro por mujeres aumenta tras alza en la participación femenina en el mundo laboral (Women's use of insurance with savings increases after rise in female participation in the labor market). La Tercera. https://www.latercera.com/pulso/noticia/contratacion-seguros-ahorro-mujeresaumenta-tras-alza-la-participacion-femenina-mundo-laboral/175010/

La Tercera (12 de mayo 2015). 76\% de los deudores morosos percibe ingresos menores a $\$ 500$ mil (76\% of delinquent debtors have an income of less than $\$ 500$ thousand). La Tercera. https://www.latercera.com/noticia/76-de-los-deudores-morosos-percibe-ingresosmenores-a-500-mil/

Lee, M. (2014). A feminist political economic critique of women and investment in the popular media. Feminist Media Studies, 14(2), 270-285. https://doi.org/10.1080/14680777.2012.728145 
Lee, M. (2019). Bubbles and Machines: Gender, Information and Financial Crises. University of Westminster Press. https://doi.org/10.16997/book34

Lee, M. \& Raesch, M. (2014). How to Study Women, Gender, and the financial Markets: A Modest Proposal for Communication Scholars. Feminist Media Studies, 14(2), 339-343. https://doi.org/10.1080/14680777.2014.887817

López, S. P. \& Bonilla, C. M. P. (2020). Financiarización e inversiones de los fondos de pensiones en la Alianza del Pacífico (Financialization and pension fund investments in the Pacific Alliance). Revista Perfiles Latinoamericanos 28(56), 207-233. https://doi.org/10.18504/pl2856-009-2020

McCombs, M. \& Valenzuela, S. (2007). The Agenda-Setting Theory. Cuadernos.info, (20), 44-50. https://doi.org/10.7764/cdi.20.111

Mendía, R. \& Pérez, C. (2020, October 2). Las caras del desempleo femenino: ¿Dónde están las mujeres? (The faces of female unemployment: Where are the women?) La Tercera. Retrieved from https://www.latercera.com/tendencias/noticia/las-caras-del-desempleofemenino-donde-estan-las-mujeres/TGSLCRN5DZCA3PCLSUBGBIRRJM/

Mizala, A. (2020, July 4). Pandemia y desigualdad de género (Pandemic and gender inequality). La Tercera. Retrieved from https://www.latercera.com/pulso/noticia/ pandemia-y-desigualdad-de-genero/DLP2IIFOTBEL3KU2M4VID6Z6Z4/

Montes, C. (19 de febrero 2019). No da lo mismo ser hombre o mujer para pedir un crédito: ellas pagan $17 \%$ más de intereses y les prestan $40 \%$ menos (It does not make a difference whether you are a man or a woman when applying for a loan: women pay $17 \%$ more interest and borrow 40\% less). La Tercera. https://www.latercera.com/que-pasa/noticia/ no-da-lo-hombre-mujer-pedir-credito-ellas-pagan-17-mas-intereses-les-prestan-40menos/526215/

Orozco, A. P. (2004). Estrategias feministas de deconstrucción del objeto de estudio de la Economía (Feminist strategies to deconstruct the object of study of the economy). Foro Interno, 4, 87-117. Retrieved from https://revistas.ucm.es/index.php/FOIN/article/view/FOIN0404110087A

Pérez-Roa, L. (2019). Consumo, endeudamiento y economía doméstica: una historia en tres tiempos para entender el estallido social (Consumption, indebtedness, and domestic economy: a three-part story to understand the social explosion). In K. Araujo (Ed.), Hilos Tensados. Para leer el octubre chileno (pp. 83 -106). Universidad de Santiago de Chile, USACH.

Pérez-Roa, L. \& Troncoso, L. (2021). Lo tuyo, lo mío y lo nuestro: hacia una lectura feminista de los arreglos económicos en pareja (Yours, mine and ours: Towards a feminist reading of economic arrangements in couples). In Proceedings of the 5th International Seminar Inequality and Social Mobility in Latin America (pp. 152-171). Retrieved from http://sedici.unlp.edu.ar/handle/10915/116358

Piñuel, J. (2002). Epistemología, metodología y técnicas de análisis de contenido (Epistemology, methodology and content analysis techniques). Estudios de Sociolingüística, 3(1), 1-42. 
Placencia, F. (2020, July 13). Efecto pandemia: 83\% de las mujeres chilenas asegura que sueldo no les alcanza para cubrir deudas (Pandemic effect: $83 \%$ of Chilean women say their salaries are not enough to cover debts ). Diario Concepción. Retrieved from https://www.diarioconcepcion.cl/economia/2020/07/13/efecto-pandemia-83-de-lasmujeres-chilenas-asegura-que-sueldo-no-les-alcanza-para-cubrir-deudas.html

Puleo A. (2007). Introducción al concepto de género (Introduction to gender concept) . In J. F. Plaza \& C. Delgado (Eds.), Género y comunicación (Gender and communication) (pp. 13-32). Fundamentos.

Raimondo Anselmino, N. (2012). Un repaso por los estudios sobre la prensa on-line en el ámbito académico nacional (A review of studies on the online press in the national academic field). Question, 1(33), 235-348. Retrieved from https://cim.unr.edu.ar/ publicaciones/2/articulos/45/un-repaso-por-los-estudios-sobre-la-prensa-online-enel-ambito-academico-nacional

Raimondo Anselmino, N. (2014). Prensa online y tipos de lectores: respuestas del público y lógicas de reconocimiento en los comentarios a las noticias del diario argentino La Nación (Online press and types of readers. Responses from the public and recognition logics in comments to the news of the Argentinian newspaper La Nación). Cuadernos.info, (34), 183-195. http://doi.org/10.7764/cdi.34.535

Roberts, A. (2015) Gender, Financial Deepening and the Production of Embodied Finance: Towards a Critical Feminist Analysis. Global Society, 29(1), 107-127. https://doi.org/10.1080/13600826.2014.975189

Roberts, A. (2016). Finance, financialization, and the production of gender. In A. A. Hozics \& J. True (Eds.), Scandalous economics: Gender and the politics of financial crises (pp. 57-99). https://doi.org/10.1093/acprof:oso/9780190204235.001.0001

Rodriguez Enriquez, C. M. (2015). Economía feminista y economía del cuidado: Aportes conceptuales para el estudio de la desigualdad (Feminist and Care Economics: Contributions conceptual framework for the study of inequality). Revista Nueva Sociedad, (256), 30-44. Retrieved from https://www.nuso.org/articulo/economia-feminista-yeconomia-del-cuidado-aportes-conceptuales-para-el-estudio-de-la-desigualdad/

Rovetto, F. (2010). La representación del trabajo de las mujeres en los medios de comunicación. El caso de las mujeres inmigrantes (The representation of women's work in the media. The case of immigrant women). Nómadas. Critical Journal of Social and Juridical Sciences, 28(4). Retrieved from https://revistas.ucm.es/index.php/NOMA/article/view/NOMA1010440113A

Rovetto, F. \& Simelio, N. (2012). Diferencias de género en los medios de comunicación. Análisis de la invisibilización del trabajo de las mujeres en la prensa española (Gender differences in the media. Analysis of the invisibilization of women's work in the Spanish press). Enfoques, 24(1), 31-52. Retrieved from https://publicaciones.uap.edu.ar/index.php/revistaenfoques/article/view/140

Rovetto, F. (2013). Percepciones sobre desigualdades de género en el trabajo periodístico (Perceptions of gender inequalities in journalism work). Global Media Journal, 10(20),54-73. Retrieved from https://biblat.unam.mx/hevila/GlobalmediajournalMexico/2013/vol10/no20/4.pdf 
Rovettto, F. \& Figueroa, L. (2019). La fuente de las desigualdades en las redacciones periodísticas. voces feministas contra la discriminación y la violencia de género en los medios (The source of inequalities in newsrooms. Feminist voices against discrimination and gender violence in the media). Revista Científica de Educación y Comunicación (18), 61-78. https://doi.org/10.25267/Hachetetepe.2019.v1.i18.7

Rudeloff, M., Brahm, T., \& Pumptow, M. (2019). Does gender matter for the use of learning opportunities? Potential explanation for the gender gap in financial literacy. Citizenship, Social and Economics Education, 18(3), 128-142. https://doi.org/10.1177/2047173419892208

SBIF. (2018). Género en el Sistema Financiero (Gender in the Financial System). Retrieved from http://www.sbif.cl/sbifweb3/internet/archivos/publicacion_12223.pdf

Villarreal, M. (2008). Sacando cuentas: prácticas financieras y marcos de calculabilidad en el México Rural (Doing the math: financial practices and calculability frameworks in rural Mexico). Revista Crítica en Desarrollo, 2, 131-149. Retrieved from http://www.idaes.edu.ar/cese/revista/Villarreal\%20Revista\%20Critica\%20No2.pdf

Wimmer, R. \& Dominik, J. (2001). Introducción a la investigación en medios masivos de comunicación (6ta ed.) (Mass media research: an introduction (6th ed.)). International Thomson Editores.

Yasinetskaya, Y. ( 27 de octubre 2017). Las mujeres en el mundo de las finanzas: una cuestión de sentido común (Women in finance: a matter of common sense). La Tercera. https://www.latercera.com/voces/las-mujeres-mundo-las-finanzas-una-cuestion-sentido-comun/

Yu, K. M., Wu, A. M., Chan, W. S., \& Chou, K. L. (2015). Gender differences in financial literacy among Hong Kong workers. Educational Gerontology, 41(4), 315-326. https://doi.org/10.1080/03601277.2014.966548

Zelizer, V. (2011). El significado social del dinero (The social meaning of money). Fondo de Cultura Económica.

\section{SOBRE LOS AUTORES}

LORENA ANDREA PÉREZ ROA, Profesora asistente de la Escuela de Trabajo Social en la Universidad de Chile e investigadora asociada de la Iniciativa del Milenio de Chile sobre Autoridad y Asimetrías de Poder. Es doctora en Ciencias Humanas por la Universidad de Montréal, magíster en Antropología por la Universidad de Chile y licenciada en Trabajo Social de la Pontificia Universidad Católica de Chile. Su investigación se centra en las relaciones socioeconómicas, las prácticas financieras de los hogares y la financiarización de la vida cotidiana.

iD https://orcid.org/0000-0002-5959-9439

SANTOS INTI ALLENDES CARO, Trabajador social, Universidad de Chile. Estudiante del magister de trabajo social en la Universidad de Chile. Tesista de la Iniciativa Milenio de Chile sobre Autoridad y Asimetrías de Poder. ANID - Programa Iniciativa Científica Milenio - Código NCS17_007(2018).

(iD) https://orcid.org/0000-0002-9190-7067 